\title{
Diferencias técnico-tácticas en taekwondistas universitarios según sexo y categoría de competición
}

\author{
Cristina MENESCARDI ${ }^{*}$, José Luis BERMEJ0 ${ }^{1}$, Carlos HERRER0 ${ }^{1}$, Isaac ESTEVAN ${ }^{1}$, Raul \\ LANDEO $^{2}$ \& Coral FALCO ${ }^{1}$ \\ ${ }^{1}$ Universidad Católica de Valencia (España) \\ ${ }^{2}$ Australian Catholic University (Australia)
}

Recepción: 02/07/2012; Aceptación: 14/08/2012; Publicación: 13/10/2012.

ARTÍCULO ORIGINAL

\section{Resumen}

Objetivo: El objetivo del presente estudio fue evaluar el comportamiento técnico-táctico, en función del sexo y la categoría de peso, de los competidores del Campeonato de España Universitario de Taekwondo de 2011.

Método: Se analizaron un total de 45 combates de las rondas finales y semifinales del campeonato, con una muestra compuesta por 30 hombres y 31 mujeres. Las variables analizadas fueron: puntuación obtenida, acciones totales, tipo de acción táctica, zona de golpeo, tipo de técnica y pierna de golpeo.

Resultados/Conclusiones: La prueba $U$ de Mann Whitney mostró diferencias significativas entre hombres y mujeres en el número total de acciones, técnicas circulares y penalizaciones. Asimismo, los resultados mostraron diferencias significativas entre sexos en la frecuencia de realización acciones circulares en la categoría de peso mosca y pesado, acciones lineales de la categoría gallo y puntuación total de la categoría de peso ligero. En la categoría de peso medio se hallaron diferencias significativas en las acciones totales, acciones de ataque, acciones al tronco, técnicas realizadas con la pierna derecha y en técnicas circulares. El comportamiento técnico-táctico de los taekwondistas es diferente tanto en función del sexo como en función del sexo y la categoría de peso, siendo las mujeres las que realizan más acciones circulares, al tronco, con la pierna derecha y totales que los hombres, mientras que estos reciben más penalizaciones que las mujeres.

Palabras clave: Taekwondo, artes marciales, deportes de combate, análisis notacional, sexo, categoría de peso.

\section{Technical-tactical differences among university level taekwondo competitors by gender and weight division}

\section{Abstract}

Aim: The aim of this study was to evaluate the technical-tactical behavior of the competitors competing in the 2011 University Taekwondo Championship of Spain.

Method: The sample consisted of 30 men and 31 women participating in 45 championship finals and semifinals combats. The variables analyzed were: the score, total number of actions, the type of tactical action, the height, the type of technic and kicking leg.

Results/Conclusions: The Mann Whitney $U$ test showed significant differences between men and women in total actions, circular techniques and warnings. The results also showed significant gender differences in circular actions in the flyweight and heavyweight categories, in the linear actions in bantamweight and total score in the lightweight category. Significant differences in total actions, attacking actions, actions to the trunk,

\section{Diferenças técnico-tácticas em praticantes de taekwondo universitários, segundo o sexo e a categoria de competição}

\section{Resumo}

Objectivo: 0 presente estudo teve como objectivo avaliar o comportamento técnico-táctico dos competidores de taekwondo do Campeonato de Espanha Universitário de 2011, em função do género e da categoria de peso.

Método: Analisou-se um total de 45 combates nas provas finais e semifinais do campeonato, com uma amostra constituída por 30 homens e 31 mulheres. As variáveis analisadas no estudo foram: pontuação obtida, acções totais, tipo de acção táctica, zona de ataque, tipo de técnica utilizada e perna de ataque.

Resultados/Conclusões:. 0 teste estatístico $U$ de Mann Whitney revela diferenças significativas entre homens e mulheres no número total de acções, técnicas circulares utilizadas e penalizações. Do mesmo modo, os resultados mostraram diferenças significativas entre os sexos na frequência de realizações circulares na categoria de peso ligeiro e pesado, acções lineares na categoria pesado e pontuação total na categoria de peso ligeiro. $\mathrm{Na}$ categoria de peso médio verificaram-se diferenças significativas nas acções totais, acções de ataque, acções ao tronco, técnicas realizadas com a perna

\footnotetext{
*E-mail: cristina.menescardi@mail.ucv.es. Dirección: Facultad de Ciencias de la Educación y del Deporte. Universidad Católica de Valencia. C/ Guillem de Castro, 175. 46008 - Valencia (España).
} 
performed techniques with right leg and circular techniques were also found in the middleweight division. The technical-tactical behavior of taekwondo competitors was found to differ between gender or gender and weight divisions. Women were found to perform more circular actions to the trunk with the right leg and total actions than men, who received more warnings than women.

Keywords: Taekwondo, martial arts, combat sports, notational analysis, gender, weight category. direita e as técnicas circulares. 0 comportamento técnico-táctico dos praticantes de taekwondo é diferente tanto em função do sexo, como em função do sexo e categoria de peso, sendo as mulheres as que realizam mais acções circulares, ao tronco, com a perna directa e totais. Os homens, no entanto, receberam mais penalizações do que as mulheres.

Palavras-chave: Taekwondo, artes marciais, desportes de combate, análise notacional, sexo, categoria de peso.

\section{1.- Introducción}

El taekwondo es un deporte de combate conocido por sus potentes y rápidos golpeos (Lystlad, Pollard, \& Graham, 2008), cuyo sistema de competición es de eliminación directa, por categorías de peso y sexo (WTF, 2012). El objetivo de los taekwondistas es vencer al oponente por puntos o KO, mediante la realización de técnicas de pierna o técnicas de brazo. Estas deben golpear en el tronco o en la cabeza (únicamente mediante técnicas de pierna) con la suficiente fuerza como para desplazar dicha parte del cuerpo y, de esta forma, conseguir puntuar (WTF, 2012). La evolución de la técnica en el taekwondo de competición ha estado, a menudo, influenciada por los cambios reglamentarios y la mejora del equipamiento, favoreciendo el uso de determinadas técnicas en detrimento de otras. Así, durante la década de los 60, se utilizaban los golpeos frontales, los bloqueos y las técnicas de puño, mientras que, los golpeos a la cabeza estaban prohibidos, con el fin de evitar lesiones. En 1967, el cambio de protectores (pasaron de ser de bambú a ser de espuma de polietileno), favoreció el uso de la patada circular con el empeine, poco utilizada hasta entonces debido a su fuerte impacto. Durante la década de los 70, y con el fin de evitar los golpeos y técnicas de puño, se introdujeron los desplazamientos, haciendo el combate más dinámico. Con la llegada de las retransmisiones deportivas, en la década de los 90, se inició una profunda revisión de las reglas de puntuación, para hacer el deporte más atractivo. Y no fue hasta el 2001 cuando se introdujo un punto adicional por la utilización de golpeos a la cabeza, pasando estos de valer uno a dos puntos, para intentar cambiar las tendencias técnico-tácticas de los competidores (Moenig, 2011).

Actualmente, un ataque válido al tronco proporciona un punto, mientras que si la técnica es realizada a la zona de la cabeza otorga tres, sumando un punto adicional si esta va precedida de una acción de giro. Por tanto, al realizar técnicas al tronco con giro previo se otorgan dos puntos, mientras que si se realizan a la altura de la cabeza con giro previo se otorgan cuatro puntos. De igual forma, existen penalizaciones que influyen en la puntuación de los competidores. Estas penalizaciones se dividen en Kyong-go (penalización de advertencia) y Gam-jeom (sanción). Dos Kyong-go sumarán un punto al rival, mientras que un único Gam-jeom también sumará un punto al adversario. Cuando un competidor sume cuatro puntos por penalizaciones, el árbitro deberá declarar al competidor perdedor por sanciones (WTF, 2012). Así pues, en el taekwondo de competición actual resulta necesario disponer de esquemas tácticos apropiados para conseguir un resultado existoso (Luk, Hong, \& Chu, 2001), que vayan de la mano con las modificaciones reglamentarias y las exigencias de la competición.

Estudios recientes muestran que las técnicas de pierna suponen la mayoría de las acciones utilizadas para puntuar (Kazemi, Casella, \& Perri, 2009a; Kazemi, Waalen, Morgan, \& White, 2006), tanto en ataque como en contraataque, entendiendo por ataque el movimiento de un deportista que inicia una secuencia, mientras que si el oponente inicia una acción para defenderse del ataque de su adversario, se considera un contraataque (González, Iglesias, Mirallas, \& Esparza, 2011b). Las acciones de ataque o contraataque han sido definidas, en función de su trayectoria, como; i) circulares, dirigidas a un lado del oponente, ii) lineales, dirigidas a la parte anterior del oponente, y finalmente iii) en giro, las cuales van precedidas de un giro mínimo de 180 (Serina \& Lieu, 1991).

El análisis técnico-táctico se ha utilizado en diversos deportes tales como squash (Hong, Robinson, \& Chan, 1998), fútbol (Thomas, Fellingham, \& Vehrs, 2009), baloncesto (Miller \& Bartlett, 
1994), rugby (Hughes \& Clarke, 1994) o tenis (O’Donoghue \& Ingram, 2001). En los deportes de combate se ha utilizado para analizar diferentes campeonatos y disciplinas deportivas, que abarcan desde los campeonatos nacionales del ámbito universitario en judo (Thomas, Fellingham, \& Vehrs, 2009) o en kárate (Laird \& McLeod, 2009), hasta los Juegos Olímpicos (Sterkowicz \& Franchini, 2000). Hasta la fecha, las investigaciones en taekwondo, publicadas en inglés o español, se han dirigido al análisis de las lesiones que sufren los deportistas (Koh, Watkinson, \& Yoon, 2004; Lystlad et al., 2008) o las características fisiológicas (Butios \& Takita, 2007) de los taekwondistas. Los estudios que han analizado el comportamiento técnico-táctico de los deportistas han centrado su atención en la distribución de frecuencias de acciones según diversas variables tales como el número total de acciones (González, Iglesias, \& Anguera, 2011a), el tipo de acción táctica aplicada (González et al., 2011a; Kazemi et al., 2009a; Kazemi et al., 2006; Matsushigue, Hartmann, \& Franchini, 2009; Santos, Franchini, \& Lima-Silva, 2011), tipo de técnica realizada (Falco, Landeo, Menescardi, Bermejo, \& Estevan, 2012; Luk et al., 2001; Matsushigue et al., 2009), zona de golpeo (Falco et al., 2012; González et al., 2011a; Luk et al., 2001), lateralidad (González et al., 2011a; Sterkowicz, Lech, Rukasz, \& Młynarczyk, 2006), así como número de acciones técnicas que obtienen puntuación (Kazemi et al., 2009a).

En general, los taekwondistas muestran una mayor tendencia a atacar (Matsushigue et al., 2009; Santos et al., 2011), utilizando en mayor media la patada circular (Koh et al., 2004; Matsushigue et al., 2009) al tronco (Falco et al., 2012; González et al., 2011a; Luk et al., 2001; Matsushigue et al., 2009), mediante la que se obtiene un punto (González et al., 2011a; Kazemi et al., 2009a). Debido a la diferencia de técnicas y acciones empleadas por los competidores en función de su peso corporal (Zen-Pin \& Ryder, 2001) el rendimiento en función de la categoría de peso de los deportistas también ha sido analizado (Falco et al., 2012; González et al., 2011a; Kazemi et al., 2009a). Sin embargo, hasta la fecha, los estudios que han evaluado el comportamiento técnicotáctico de los taekwondistas han asumido una conducta similar en hombres y mujeres (Cular et al., 2011; Kazemi et al., 2009a; Kazemi et al., 2006; Matsushigue et al., 2009; Sterkowicz et al., 2006), o bien han observado un patrón en hombres (González et al., 2011a; Luk et al., 2001; Santos et al., 2011) o mujeres (Wąsik \& Slęzak, 2004) de forma separada, sin llegar a comparar ambos sexos o la conveniencia de estudiarlos por separado. En el ámbito de la biomecánica, existe evidencia empírica (Pieter \& Pieter, 1995) que muestra diferencias en cuanto a la velocidad de ejecución de los golpeos, lo cual podría influir en la preferencia por unas técnicas de pierna en función del sexo.

A partir de estas consideraciones, el objetivo del presente estudio es comparar el comportamiento tecnico-tactico de los competidores del Campeonato de España Universitario de Taekwondo de 2011 en función del sexo y la categoria de peso, atendiendo a: i) la puntuación obtenida, ii) acciones totales, iii) tipo de acción táctica, iv) zona de golpeo, v) tipo de técnica y vi) pierna de golpeo utilizada durante los combates. Estas variables permitirán explicar el comportamiento técnico-táctico del taekwondista en competición, tanto en hombres como en mujeres, y en función de las correspondientes categorías de peso dentro de cada sexo. Este análisis conjunto por sexo y categoría de competición se ajusta más a la realidad de la competición, ya que los atletas compiten dentro de un mismo sexo (hombre o mujer) y posteriormente, dentro de una categoría determinada de acuerdo al peso de cada competidor. Este análisis a su vez podría proveer a los entrenadores y deportistas de directrices técnico-tácticas para la orientación eficaz de los entrenamientos y desarrollar al máximo sus posibilidades, así como para que puedan buscar estrategias con las que hacer frente a potenciales adversarios.

\section{2.- Método}

\section{1.- Muestra}

La muestra estuvo compuesta por la totalidad $(N=61)$ de los finalistas y semifinalistas del Campeonato de España Universitario de Taekwondo 2011, hombres $(n=30)$ y mujeres $(n=31)$ cuya edad media fue de 22,39 años $(D T=9,10)$. Todos los deportistas participaron voluntariamente en el estudio y firmaron un documento de consentimiento informado donde se indicaba el objeto del estudio y la utilización de las filmaciones con fines científicos. 
Se analizaron un total de 45 combates pertenecientes a las finales y semifinales del campeonato. El análisis se hizo en las siguientes categorías de peso: minimosca (3 semifinales y 2 finales), mosca (4 semifinales y 2 finales), gallo (4 semifinales y 2 finales), pluma ( 3 semifinales y 2 finales), ligero ( 4 semifinales y 2 finales), superligero ( 4 semifinales y 2 finales), medio (4 semifinales y 2 finales) y pesado (3 semifinales y 2 finales) ${ }^{1}$. Tres semifinales, dos de ellas masculinas (pluma y pesado) y una femenina (minimosca) no se realizaron por lesión de uno de los participantes.

\section{2.- Procedimiento}

El análisis del comportamiento técnico-táctico de los medallistas del Campeonato de España Universitario de Taekwondo 2011 fue de tipo descriptivo y diferencial. La muestra se agrupó en función de la categoría de peso y el sexo de los participantes. Las variables analizadas fueron la puntuación obtenida (puntuación total, acciones de 1 punto, 2 puntos, 3 puntos o 4 puntos, y penalizaciones), la frecuencia de realización de acciones totales, acciones tácticas (ataque o contraataque), zona de golpeo (cabeza o tronco), tipo de técnica según la trayectoria utilizada (lineal, circular o con giro) y pierna de golpeo (derecha o izquierda). Sobre la base de estudios previos (Kazemi et al., 2009a; Kazemi et al., 2006; Matsushigue et al., 2009; Santos et al., 2011), el análisis de las filmaciones se ejecutó con una metodología observacional de único observador. Para tal efecto, se formó al observador durante un período de 40 horas siguiendo las fases de entrenamiento sugeridas por Anguera (2003). Para el visionado de los videos se utilizó el software Dartfish TeamPro v.6.0 (Dartfish, Fribourg, Switzerland).

\section{3.- Análisis estadístico}

La prueba Kolmogorov-Smirnov indicó que la muestra no seguía una distribución normal, por lo que se utilizó la prueba no paramétrica de Mann Whitney para el análisis diferencial del comportamiento técnico-táctico utilizado por los deportistas en función del sexo, primero, y después en función del sexo y la categoría de peso. La fiabilidad de los datos fue determinada a partir del análisis de cinco combates seleccionados al azar (Salvador, Suay, Martinez-Sanchis, Simon, \& Brain, 1999), tras el entrenamiento del observador, de los cuales se obtuvo el índice Kappa para determinar la concordancia entre dos observadores, el investigador y un experto, con un valor de 0,96 y el coeficiente de correlación intraclase (CCI), para determinar la concordancia intra-observador con un valor de $0,85(p<0,01)$, que revela que el análisis realizado en este estudio fue altamente fiable (Blázquez, 1990). Todo ello se llevó a cabo con el paquete estadístico SPSS versión 17.0 (Chicago, Inc.) licencia de la Universidad Católica de Valencia, aceptándose un nivel de significación de $p<0,05$.

\section{3.- Resultados}

En la Tabla 1 se pueden observar los análisis descriptivos (media y desviación típica) y diferenciales de las variables analizadas.

La prueba $U$ de Mann Whitney arrojó diferencias significativas entre hombres y mujeres en el número total de acciones $(U=764,00 ; p=0,04)$, en las técnicas circulares $(U=688,50 ; p=0,01)$ y en las penalizaciones $(U=824,00 ; p=0,01)$. Tal y como muestra la Tabla 1 , no se hallaron diferencias significativas en el resto de variables.

La Tabla 2 muestra la comparación de las diferentes variables en función del sexo y categoría de peso. La prueba $U$ de Mann Whitney mostró diferencias significativas, entre hombres y

\footnotetext{
${ }^{1}$ Pesos correspondientes a las categorías de competición minimosca: < 54 kg en hombres y < 46 kg en mujeres; mosca: 54 - $58 \mathrm{~kg}$ en hombres y 46 - $49 \mathrm{~kg}$ en mujeres; gallo: 58 - $63 \mathrm{~kg}$ en hombres y 49 - $53 \mathrm{~kg}$ en mujeres; pluma: 63 - $68 \mathrm{~kg}$ en hombres y 53 - $57 \mathrm{~kg}$ en mujeres; ligero: 68 - $74 \mathrm{~kg}$ en hombres y 57 - $62 \mathrm{~kg}$ en mujeres; superligero: $74-80 \mathrm{~kg}$ en hombres y 62 - $67 \mathrm{~kg}$ en mujeres; medio: 80 - $87 \mathrm{~kg}$ en hombres y $67-73 \mathrm{~kg}$ en mujeres; pesado: > $87 \mathrm{~kg}$ en hombres y > $73 \mathrm{~kg}$ en mujeres.
} 
mujeres en la frecuencia de realización acciones circulares en la categoría de peso mosca $(U=2,0 ; p$ $=0,01)$ y pesado $(U=0,5 ; p=0,01)$, en las acciones lineales de la categoría gallo $(U=1,5 ; p=0,01) \mathrm{y}$ en la puntuación total de la categoría de peso ligero $(U=4,0 ; p=0,03)$. En la categoría de peso medio se hallaron diferencias significativas en las acciones totales $(U=1,0 ; p=0,04)$, las acciones de ataque $(U=2,0 ; p=0,01)$, las acciones al tronco $(U=1,0 ; p=0,04)$, las técnicas realizadas con la pierna derecha $(U=5,0 ; p=0,04)$ y en las técnicas circulares $(U=1,0 ; p=0,01)$. Sin embargo, no se hallaron diferencias significativas en el resto de parámetros analizados.

Tabla 1

Análisis descriptivo y diferencial de la totalidad de competidores

\begin{tabular}{cccccccc}
\hline & \multicolumn{3}{c}{ Hombres $(n=30)$} & & \multicolumn{3}{c}{ Mujeres $(n=31)$} \\
\cline { 2 - 5 } \cline { 7 - 8 } & $M$ & $D T$ & $\%$ & & $M$ & $D T$ & $\%$ \\
\hline Puntuación total & 4,45 & 2,69 & 100,00 & & 4,55 & 3,55 & 100,00 \\
Acciones 1Pto & 3,16 & 2,01 & 90,03 & & 3,78 & 3,20 & 84,66 \\
Acciones 2Ptos & 0,05 & 0,21 & 1,42 & & 0,00 & 0,00 & 0,00 \\
Acciones 3Ptos & 0,30 & 0,55 & 8,55 & & 0,20 & 0,58 & 15,34 \\
Acciones 4Ptos & 0,00 & 0,00 & 0,00 & & 0,00 & 0,00 & 0,00 \\
Penalizaciones & $0,27^{*}$ & 0,54 & 100,00 & & $0,04^{*}$ & 0,20 & 100,00 \\
Acciones totales & $31,52^{*}$ & 7,87 & 100,00 & & $34,72^{*}$ & 12,81 & 100,00 \\
Contraataques & 7,70 & 4,47 & 24,43 & & 9,13 & 5,73 & 26,30 \\
Ataques & 23,82 & 7,64 & 75,57 & & 25,59 & 10,98 & 73,70 \\
Zona de golpeo & & & & & & & \\
Tronco & 28,25 & 7,37 & 89,63 & & 31,50 & 11,74 & 90,73 \\
Cabeza & 3,27 & 2,44 & 10,37 & & 3,22 & 2,84 & 9,27 \\
Tipo de técnica & & & & & & & \\
Lineales & 7,98 & 5,57 & 25,32 & & 6,48 & 4,07 & 18,66 \\
Circulares & $21,45^{*}$ & 7,37 & 68,05 & & $26,50 *$ & 11,07 & 76,32 \\
Giro & 2,09 & 2,20 & 6,63 & & 6,63 & 1,74 & 5,02 \\
Lateralidad & & & & & & & \\
Derecha & 16,00 & 7,38 & 50,76 & & 18,46 & 11,18 & 53,17 \\
Izquierda & 15,52 & 8,10 & 49,24 & & 16,26 & 9,20 & 46,83 \\
\hline
\end{tabular}

Nota: * diferencias significativas $(p<0,05)$ entre sexos.

\section{4.- Discusión}

El objetivo del presente estudio fue analizar el comportamiento técnico-táctico de los medallistas del Campeonato de España Universitario de Taekwondo 2011 en función del sexo y categoría de peso de los competidores. Dicho comportamiento ha sido analizado sobre la base de: i) la puntuación obtenida, ii) las acciones totales, iii) el tipo de acción táctica, iv) la zona de golpeo, v) tipo de técnica y vi) lateralidad utilizada durante los combates.

En función del sexo, los resultados mostraron un promedio superior de acciones totales por combate en mujeres que en hombres. Sin embargo, no arrojaron diferencias en la puntuación obtenida. Es decir, las mujeres realizaron un mayor número de acciones pero no por ello fueron más eficaces. Asimismo, el número de acciones totales resultó ser superior a los encontrados en otros estudios sobre competiciones de nivel internacional (González et al., 2011a; Santos et al., 2011), mientras que se asemejan a los encontrados en estudios sobre competiciones de nivel nacional (Matsushigue et al., 2009). Parece ser que, a medida que los taekwondistas participan en competiciones de mayor nivel, el número de técnicas que realizan es menor. Ello podría ser atribuido a una menor conciencia táctica y comportamiento reflexivo en los deportistas de nivel universitario y nacional en comparación con los deportistas internacionales (Santos et al., 2011). Por otra parte y en la línea de estudios previos (Kazemi et al., 2006; Kazemi et al., 2009a), los resultados de nuestro estudio muestran que los hombres son penalizados en mayor medida que las mujeres, lo que podría sugerir formas de combate más impulsivas por parte de los competidores masculinos (Kazemi et al., 2006). 


\section{Tabla 2}

Análisis descriptivo y diferencial de las diferentes categorías de competición en función del sexo

\begin{tabular}{|c|c|c|c|c|c|c|c|c|c|c|c|c|c|c|c|c|}
\hline & & & & Hombres & & & & & & & & & Mujeres & & & \\
\hline & $\begin{array}{c}\text { Minimosca } \\
(n=6)\end{array}$ & $\begin{array}{c}\text { Mosca } \\
(n=6)\end{array}$ & $\begin{array}{c}\text { Gallo } \\
(n=6)\end{array}$ & $\begin{array}{l}\text { Pluma } \\
(n=4)\end{array}$ & $\begin{array}{l}\text { Ligero } \\
(n=6) \\
\end{array}$ & $\begin{array}{l}\text { Superlige } \\
\text { ro }(n=6)\end{array}$ & $\begin{array}{l}\text { Medio } \\
(n=6)\end{array}$ & $\begin{array}{c}\text { Pesado } \\
(n=4)\end{array}$ & $\begin{array}{c}\text { Minimosca } \\
(n=4)\end{array}$ & $\begin{array}{l}\text { Mosca } \\
(n=6)\end{array}$ & $\begin{array}{c}\text { Gallo } \\
(n=6)\end{array}$ & $\begin{array}{l}\text { Pluma } \\
(n=6)\end{array}$ & $\begin{array}{l}\text { Ligero } \\
(n=6)\end{array}$ & $\begin{array}{c}\text { Superligero } \\
(n=6)\end{array}$ & $\begin{array}{l}\text { Medio } \\
(n=6)\end{array}$ & $\begin{array}{l}\text { Pesado } \\
(n=4) \\
\end{array}$ \\
\hline & $M \pm D T$ & $M \pm D T$ & $M \pm D T$ & $M \pm D T$ & $M \pm D T$ & $M \pm D T$ & $M \pm D T$ & $M \pm D T$ & $M \pm D T$ & $M \pm D T$ & $M \pm D T$ & $M \pm D T$ & $M \pm D T$ & $M \pm D T$ & $M \pm D T$ & $M \pm D T$ \\
\hline Puntuación total & l $3,83 \pm 2,32$ & $5,50 \pm 2,17$ & $3,67 \pm 1,86$ & $4,00 \pm 2,58$ & $5,67 \pm 3,33^{*}$ & $3,00 \pm 2,53$ & $5,33 \pm 4,03$ & $4,50 \pm 2,38$ & $1,75 \pm 0,96$ & $6,50 \pm 3,83$ & $3,50 \pm 2,43$ & $3,50 \pm 3,00$ & $4,00 \pm 2,82^{*}$ & $2,83 \pm 4,11$ & $4,17 \pm 1,94$ & $8,50 \pm 3,78$ \\
\hline 1Pto & $2,83 \pm 1,94$ & $4,50 \pm 2,35$ & $2,17 \pm 1,60$ & $3,25 \pm 1,71$ & $0,50 \pm 0,84$ & $0,00 \pm 0,00$ & $0,50 \pm 0,84$ & $0,25 \pm 0,50$ & $1,75 \pm 0,96$ & $4,83 \pm 1,72$ & $3,00 \pm 2,83$ & $3,50 \pm 3,00$ & $0,00 \pm 0,00$ & $0,00 \pm 0,00$ & $0,17 \pm 0,40$ & $0,67 \pm 1,21$ \\
\hline 2Ptos & $0,00 \pm 0,00$ & $0,00 \pm 0,00$ & $0,00 \pm 0,00$ & $0,00 \pm 0,00$ & $0,00 \pm 0,00$ & $0,00 \pm 0,00$ & $0,00 \pm 0,00$ & $0,00 \pm 0,00$ & $0,00 \pm 0,00$ & $0,00 \pm 0,00$ & $0,00 \pm 0,00$ & $0,00 \pm 0,00$ & $0,00 \pm 0,00$ & $0,00 \pm 0,00$ & $0,00 \pm 0,00$ & $0,00 \pm 0,00$ \\
\hline 3Ptos & $0,33 \pm 0,52$ & $0,17 \pm 0,41$ & $0,33 \pm 0,52$ & $0,25 \pm 0,50$ & $3,17 \pm 1,72$ & $2,83 \pm 2,71$ & $3,50 \pm 2,59$ & $3,00 \pm 0,82$ & $0,00 \pm 0,00$ & $0,50 \pm 0,84$ & $0,17 \pm 0,41$ & $0,00 \pm 0,00$ & $4,00 \pm 2,82$ & $2,50 \pm 4,32$ & $3,50 \pm 2,25$ & $6,50 \pm 4,63$ \\
\hline 4Ptos & $0,00 \pm 0,00$ & $0,00 \pm 0,00$ & $0,00 \pm 0,00$ & $0,00 \pm 0,00$ & $0,17 \pm 0,41$ & $0,00 \pm 0,00$ & $0,00 \pm 0,00$ & $0,25 \pm 0,50$ & $0,00 \pm 0,00$ & $0,00 \pm 0,00$ & $0,00 \pm 0,00$ & $0,00 \pm 0,00$ & $0,00 \pm 0,00$ & $0,00 \pm 0,00$ & $0,00 \pm 0,00$ & $0,00 \pm 0,00$ \\
\hline Penalizaciones & s $0,00 \pm 0,00$ & $0,50 \pm 0,84$ & $0,33 \pm 0,52$ & $0,00 \pm 0,00$ & $0,83 \pm 0,75$ & $0,00 \pm 0,00$ & $0,17 \pm 0,41$ & $0,25 \pm 0,50$ & $0,00 \pm 0,00$ & $0,17 \pm 0,41$ & $0,00 \pm 0,00$ & $0,00 \pm 0,00$ & $0,00 \pm 0,00$ & $0,00 \pm 0,00$ & $0,17 \pm 0,40$ & $0,00 \pm 0,00$ \\
\hline Acciones totales & $29,00 \pm 4,86$ & $34,83 \pm 4,79$ & $30,33 \pm 5,57$ & $32,00 \pm 4,90$ & $41,33 \pm 11,04$ & $27,17 \pm 5,27$ & $29,00 \pm 8,05^{*}$ & $27,25 \pm 8,54$ & $20,00 \pm 7,79$ & $37,50 \pm 6,89$ & $33,33 \pm 8,09$ & $19,50 \pm 14,52$ & $238,33 \pm 11,67$ & $33,83 \pm 9,34$ & $50,00 \pm 8,43^{*}$ & $40,33 \pm 5,12$ \\
\hline Contraataque & $5,83 \pm 3,66$ & $8,33 \pm 5,05$ & $7,00 \pm 2,53$ & $7,75 \pm 3,77$ & $10,17 \pm 4,26$ & $6,83 \pm 5,56$ & $8,83 \pm 6,52$ & $6,50 \pm 4,12$ & $4,50 \pm 3,11$ & $8,83 \pm 3,54$ & $9,67 \pm 5,05$ & $6,00 \pm 7,46$ & $10,83 \pm 3,54$ & $6,17 \pm 4,53$ & $12,83 \pm 5,23$ & $12,67 \pm 7,81$ \\
\hline Ataque & $23,17 \pm 5,12$ & $26,50 \pm 8,22$ & $23,33 \pm 5,89$ & $24,25 \pm 4,57$ & $31,17 \pm 10,30$ & $20,33 \pm 7,28$ & $20,17 \pm 5,49 *$ & $20,75 \pm 9,98$ & $15,50 \pm 10,21$ & $28,67 \pm 5,85$ & $23,67 \pm 9,75$ & $13,50 \pm 10,23$ & $327,50 \pm 9,83$ & $27,67 \pm 8,50$ & $37,17 \pm 9,36^{*}$ & $27,67 \pm 9,22$ \\
\hline \multicolumn{17}{|l|}{ Zona golpeo } \\
\hline Tronco & $25,00 \pm 4,69$ & $31,50 \pm 5,82$ & $27,33 \pm 6,59$ & $27,25 \pm 4,72$ & $36,67 \pm 10,03$ & $25,00 \pm 5,37$ & $26,33 \pm 7,15^{*}$ & $25,75 \pm 8,18$ & $18,25 \pm 7,37$ & $33,00 \pm 4,20$ & $30,00 \pm 6,90$ & $18,00 \pm 13,84$ & $434,00 \pm 10,37$ & $30,83 \pm 8,56$ & $47,00 \pm 6,51^{*}$ & $36,50 \pm 6,09$ \\
\hline Cabeza & $4,00 \pm 2,28$ & $3,33 \pm 1,97$ & $3,00 \pm 2,45$ & $4,75 \pm 3,40$ & $4,67 \pm 1,37$ & $2,17 \pm 3,37$ & $2,67 \pm 2,42$ & $1,50 \pm 1,29$ & $1,75 \pm 1,26$ & $4,50 \pm 3,27$ & $3,33 \pm 2,50$ & $1,50 \pm 1,87$ & $4,33 \pm 3,14$ & $3,00 \pm 2,96$ & $3,00 \pm 4,05$ & $3,83 \pm 2,63$ \\
\hline \multicolumn{17}{|l|}{ Lateralidad } \\
\hline Derecha & $13,50 \pm 4,89$ & $16,33 \pm 3,93$ & $16,33 \pm 8,98$ & $10,25 \pm 9,29$ & $23,67 \pm 7,76$ & $16,50 \pm 4,23$ & $11,83 \pm 8,04^{*}$ & $18,50 \pm 6,35$ & $7,25 \pm 6,45$ & $17,33 \pm 5,82$ & $21,83 \pm 9,45$ & $10,83 \pm 9,37$ & $22,50 \pm 14,15$ & $14,00 \pm 8,74$ & $29,00 \pm 13,94^{*}$ & * $21,17 \pm 6,85$ \\
\hline Izquierda & $15,50 \pm 4,93$ & $18,50 \pm 5,58$ & $14,00 \pm 11,93$ & $21,75 \pm 7,63$ & $17,67 \pm 9,07$ & $10,67 \pm 8,64$ & $17,17 \pm 6,94$ & $8,75 \pm 3,77$ & $12,75 \pm 7,18$ & $20,17 \pm 8,77$ & $11,50 \pm 6,09$ & $8,67 \pm 6,68$ & $15,83 \pm 7,83$ & $19,83 \pm 9,94$ & $21,00 \pm 12,74$ & - $19,17 \pm 8,51$ \\
\hline \multicolumn{17}{|l|}{ Tipo técnica } \\
\hline Lineal & $10,33 \pm 5,75$ & $13,67 \pm 6,31$ & $3,83 \pm 2,40^{*}$ & $13,00 \pm 4,24$ & $8,83 \pm 5,91$ & $4,50 \pm 2,59$ & $4,17 \pm 3,25$ & $6,75 \pm 2,99$ & $3,25 \pm 2,06$ & $5,67 \pm 2,50$ & $9,00 \pm 4,05^{*}$ & $4,17 \pm 5,71$ & $8,33 \pm 5,92$ & $6,33 \pm 2,73$ & $7,83 \pm 3,65$ & $6,17 \pm 2,48$ \\
\hline Circular & $15,83 \pm 7,11$ & $19,50 \pm 5,24^{*}$ & $24,00 \pm 6,39$ & $17,00 \pm 5,35$ & $30,33 \pm 8,52$ & $20,00 \pm 4,52$ & $23,67 \pm 6,35^{*}$ & $19,00 \pm 6,38^{*}$ & $15,50 \pm 5,74$ & $28,83 \pm 3,71^{*}$ & 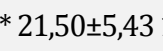 & $12,67 \pm 10,27$ & $729,17 \pm 10,83$ & $26,17 \pm 8,13$ & $41,17 \pm 7,57^{*}$ & $33,33 \pm 3,07^{*}$ \\
\hline Giro & $2,83 \pm 3,82$ & $1,50 \pm 0,55$ & $1,67 \pm 2,42$ & $0,75 \pm 0,96$ & $1,83 \pm 1,94$ & $1,50 \pm 2,35$ & $0,67 \pm 0,82$ & $1,25 \pm 1,50$ & $1,25 \pm 1,50$ & $3,33 \pm 3,67$ & $3,50 \pm 3,27$ & $3,50 \pm 2,35$ & $1,17 \pm 1,16$ & $2,50 \pm 2,73$ & $1,50 \pm 1,22$ & $1,00 \pm 1,26$ \\
\hline
\end{tabular}

Nota: * diferencias significativas $(p>0,05)$ entre sexos dentro de la misma categoría de competición. 
Otro objetivo de nuestro estudio fue el de establecer si existieron diferencias entre hombres y mujeres teniendo en cuenta su categoría de peso. En cuanto a la puntuación, en la categoría ligero los hombres obtuvieron más puntos por combate que las mujeres. En la misma línea, Kazemi et al. (2009a) muestran que los hombres realizan un mayor número de técnicas eficaces que sus homólogas femeninas. Sin embargo, no hemos encontrado más investigaciones que comparen la puntuación obtenida por hombres y mujeres en función de la categoría de peso, y por tanto, serían necesarios más estudios de ésta índole para confirmar esta tendencia.

En cuanto al número de técnicas realizadas, únicamente se hallaron diferencias en la categoría de peso medio, siendo las mujeres las que realizan mayor número de acciones de ataque que los hombres. Aunque no hay estudios hasta la fecha que comparen las acciones técnico-tácticas en función de la categoría de peso y sexo de los deportistas, estos resultados se asemejan a estudios previos que muestran que las mujeres realizan un mayor número de acciones ofensivas que los hombres (Kazemi et al., 2006). En relación a las acciones tácticas, y en consonancia con estudios previos (Matsuhique et al., 2009; Santos et al., 2011), los taekwondistas, en general, utilizan más acciones de ataque que de contraataque para ganar el combate. En esta línea, Yujin y Zeng (1999) sugieren que el entrenamiento de los atletas debe estar motivado por atacar en lugar de contraatacar. Nuestros resultados difieren de los hallados por estudios más recientes (Cular et al., 2011) que muestran una predominancia de las acciones de contraataque. Esta diferencia entre los datos hallados podría deberse a que los deportistas universitarios tienden a suplir su inexperiencia con acciones más agresivas. Sin embargo, los resultados del presente estudio no se pueden relacionar con la cantidad de puntos que se obtuvieron a partir de acciones específicas. Futuros estudios deberían identificar el tipo de acciones técnico-tácticas que se utiliza para puntuar.

Independientemente de la acción táctica realizada, se puede golpear al oponente con cualquier tipo de técnica (p.ej., lineal, circular o con giro) (Serina \& Lieu, 1991). En la línea de estudios previos (González et al., 2011a; Koh et al., 2004; Luk et al., 2001; Matsuhique et al., 2009; Wąsik \& Slęzak, 2004), en nuestro estudio hay una preferencia por realizar acciones circulares frente a las acciones lineales o con giro. Lo cual implica que la puntuación establecida reglamentariamente, donde las acciones con giro previo pueden lograr un punto adicional, no influye en la utilización de este tipo de técnicas. Del mismo modo, un menor tiempo de ejecución de las técnicas lineales frente al resto (Lee et al., 2005) no conlleva una mayor realización de las mismas. Dicha preferencia por las técnicas circulares podría deberse a su alta velocidad y precisión (Pieter \& Pieter, 1995) por lo que han acabado imponiéndose frente a la potencia de las patadas frontales, características de los primeros años de competición (Moenig, 2011). Los resultados muestran diferencias en la realización de acciones circulares entre ambos sexos, siendo las mujeres las que realizan un número mayor que los hombres, y, específicamente, en las categorías de peso de mosca, medio y pesado, mientras que las mujeres de gallo realizaron más acciones lineales que los hombres gallo. Esta diferencia podría atribuirse a la tardía incorporación de la mujer en este deporte en comparación con el hombre (Kazemi et al., 2006), lo cual conllevaría a competidores con menos experiencia, a compensar con mayor agresividad (Kazemi et al., 2009b), lo cual podría explicar por qué las competidoras realizan un número mayor de acciones que los hombres.

En las técnicas de pierna de taekwondo se ven implicados tanto los miembros dominantes como los no dominantes (Tang, Chang, \& Nien, 2007). Los resultados de nuestro estudio no muestran una lateralidad predominante ni en función del sexo, ni en función del sexo y categoría de peso, salvo en la categoría de peso medio donde las mujeres realizan un mayor número de acciones con pierna derecha que los hombres. Esto coincide con estudios previos, que no muestran diferencias significativas entre las piernas dominantes y no dominantes, tanto en investigaciones del ámbito de la biomecánica (Tang et al., 2007) como notacionales (González et al., 2011a). El hecho de ser capaz de golpear al contrario con cualquier pierna se considera una ventaja dentro de la élite del taekwondo, ya que tener una habilidad bilateral permite atacar y contraatacar al contrario sin que este pueda predecir con facilidad el lado por el que recibirá el ataque o el contraataque (Tang et al., 2007). Investigaciones previas (Falco et al., 2012) ya mostraron que la categoría medio femenino también realiza un mayor número de acciones que sus homólogas de diferentes pesos. Por tanto, podemos atribuir nuestros resultados al hecho de que estas competidoras realizaron más acciones totales y técnicas circulares que los hombres. 
En cuanto a la zona de golpeo, los competidores prefieren realizar las acciones al tronco en lugar de a la cabeza. Esto coincide con estudios que sugieren que los golpeos se dirigen, en mayor medida, a la zona del tronco (Cular et al., 2011; González et al., 2011a; Luk et al., 2001). Sin embargo, estudios en contra muestran que los deportistas preferirían golpear a la cabeza del oponente debido a que la puntuación obtenida sería mayor que si la acción se realiza al tronco (Koh et al., 2004), puesto que no hay diferencias temporales en la realización de acciones circulares al tronco o a la cabeza (Estevan, Molina-García, Falco, \& Álvarez, 2010; Falco, Estevan, Menescardi, \& Ruiz, 2011a). Nuestros resultados no muestran diferencias en la realización de golpeos a diferentes alturas para cada sexo, lo cual concuerda con las investigaciones previas que muestran datos relativos al tiempo de ejecución similares entre hombres $(0,740 \pm 0,086 \mathrm{~s})$ (Falco, Estevan, \& Vieten, 2011b) y mujeres (0,770 $\pm 0,030 \mathrm{~s})$ (Estevan, Herrero, Bermejo, Menescardi, \& Falco, 2012). En cuanto a las diferencias en función del sexo y categoría de peso, sí se hallaron diferencias entre competidores de diferente sexo dentro de la misma categoría de peso, siendo las mujeres de peso medio las que realizaron más acciones al tronco que los hombres. Esta preferencia por realizar acciones al tronco podría deberse a que hay una menor actividad en diversos grupos musculares de la pierna al realizar acciones al tronco en comparación que las acciones realizadas a la cabeza (Luk \& Hong, 2000, citado por Luk et al., 2001). Por ello, una mayor ejecución de acciones al tronco equivaldría a un menor grado de fatiga durante la competición (Luk et al., 2001) de las competidoras, las cuales tienen una menor capacidad aeróbica y anaeróbica que los hombres (Heller et al., 1998).

La revisión de la literatura actual sugiere que este es el primer estudio que analiza el tipo de técnica utilizada, la lateralidad y la zona de golpeo utilizada en función del sexo y categoría de peso. Del mismo modo, este es el primer estudio tras los cambios reglamentarios de 2010, cuando se modificaron las puntuaciones establecidas para los diferentes golpeos; sin embargo, no se han analizado las acciones utilizadas para defender una acción puntuable tales como los desplazamientos o bloqueos, los cuales podrían explicar en mayor medida la eficacia de las acciones. Futuros estudios son necesarios para confirmar si se mantienen estas tendencias con la nueva reglamentación, de igual forma que se deberían abarcar los parámetros comentados para un mayor conocimiento del rendimiento en este deporte. Por último, cabría señalar la cautela a la hora de generalizar los datos que se ofrecen, ya que solo podrían ser aplicables a la población universitaria española finalista y semifinalista, puesto que no se ha tenido en cuenta la totalidad de los participantes. Pese a estas limitaciones, estos datos pueden proporcionar una mejor compresión del comportamiento técnico-táctico en los combates de taekwondo y constituyen una base para futuras investigaciones.

\section{5.- Conclusiones y aplicaciones prácticas}

Según los resultados de esta investigación se pueden establecer las siguientes conclusiones: porcentualmente, los taekwondistas universitarios, tanto hombres como mujeres, utilizan preferentemente acciones de ataque mediante técnicas circulares al tronco, sin preferencia de lateralidad, con las que consiguen un punto al impactar en el oponente. Sin embargo, los taekwondistas universitarios tienen un comportamiento técnico-táctico diferente en función del sexo, siendo las mujeres las que realizan más acciones circulares, al tronco y con pierna derecha y por tanto, más acciones totales que los hombres, mientras que estos reciben más penalizaciones que las mujeres. Por ello, el entrenamiento de ambos sexos debe ser diferente. De igual forma, dentro de la misma categoría de peso también se produce un comportamiento diferente entre sexos, siendo generalmente las mujeres las que realizan un mayor número de acciones que los hombres. En estas categorías, una mayor frecuencia de acciones podría conllevar una reorientación del entrenamiento físico de las deportistas con vistas a soportar mejor las exigencias de la competición.

\section{Agradecimientos}

La presente investigación ha sido financiada por la Universidad Católica de Valencia (UCV 2011 -007 -002). Queremos agradecer a Darthfish y la Real Federación Española de Taekwondo su generosa colaboración para la obtención de los videos y programa. 


\section{Referencias}

Anguera, M. T. (2003). La observación. En C. Moreno Rosset (ed.), Evaluación psicológica. Concepto, proceso y aplicación en las áreas del desarrollo y de la inteligencia (pp. 271-308). Madrid: Sanz y Torres.

Blázquez, D. (1990). Evaluar en educación física. Barcelona: Inde.

Butios, S., \& Takita, N. (2007). Changes in heart rate and blood lactate concentration as intensity parameters during simulated Taekwondo competition. Journal of Sports Medicine and Physical Fitness, 47, 179-185.

Cular, D., Krstulovic, S., \& Tomljanovic, M. (2011). The differences between medal winners and nonwinnwers at the 2008 Olympic Games taekwondo tournament. Human Movement, 12(2), 165170.

Estevan, I., Herrero, C., Bermejo, J. L., Menescardi, C., \& Falco, C. (2012). Análisis cinemático en función del tipo de patada en Taekwondo. En VVAA. (eds.), I Seminario Científico Internacional de la Red Universitaria Euro-Americana de Actividad Física, Educación Física, Deporte y Recreación (pp. 11-19). Universidad de Murcia, Murcia, España.

Estevan, I., Molina-García, J., Falco, C., \& Álvarez, O. (2010). Comparación de la eficiencia de la patada circular al pecho y a la cara en taekwondo según la distancia de ejecución. RICYDE. Revista Internacional de Ciencias del Deporte, 6(21), 269-279.

Falco, C., Estevan, I., Menescardi, C., \& Ruiz, L. (2011a). The roundhouse Kick in function of the two target level. En A. A. Figueiredo, \& C. Gutiérrez-García (eds.), Scientific Congress on Martial Arts and Combat Sports (pp. 36-38). Associaçao para o desenvolvimiento e investigaçao de Viseu, Viseu, Portugal.

Falco, C., Estevan, I., \& Vieten, M. (2011b). Kinematical analysis of five different kicks in Taekwondo. Portuguese Journal of Sport Sciences, 11(2), 219 - 222.

Falco, C., Landeo, R., Menescardi, C., Bermejo, J. L., \& Estevan, I. (2012). Match analysis in a University Taekwondo Championship. Advances in Physical Education, 2(1), 28-31.

González, C., Iglesias, X., \& Anguera, M. (2011a). Tactical moves in top level competition taekwondo combat. A descriptive study. En A. A. Figueiredo, \& C. Gutiérrez-García (eds.), Scientific Congress on Martial Arts and Combat Sports (pp. 48-49). Associaçao para o desenvolvimiento e investigaçao de Viseu, Viseu, Portugal.

González, C., Iglesias, X., Mirallas, J., \& Esparza, G. (2011b). Sistematizació de l'acció tàctica en el taekwondo d'alta competició. Apunts: Educació Física i Esports, 103(1), 56-67.

Heller, J., Peric, T., Dlouha, R., Kohlikova, E., Melichna, J., \& Novakova, H. (1998). Physiological profiles of male and female taekwon-do (ITF) black belts. Journal of Sports Science, 16, 243249.

Hong, Y., Robinson, P. D., \& Chan, W. K. (1998). Game strategy used by the world's top female squash players in international competitions - A notational analysis. Women in Sport and Physical Activity Journal, 7(1), 27-44.

Hughes, M., \& Clarke, A. (1994). Computerized notation analysis of rugby union to examine the effects of law changes upon the patterns of play by the international tennis. Journal of Sports Sciences, 12(2), 180.

Kazemi, M., Casella, C., \& Perri, G. (2009a). 2004 Olympic Tae Kwon Do Athlete Profile. The Journal of the Canadian Chiropractic Association, 53(2), 144-152.

Kazemi, M., Chudolinski, A., Turgeon, M., Simon, A., Ho, E., \& Coombe, L. (2009b). Nine year longitudinal retrospective study of Taekwondo injuries. Journal of Canadian Chiropractic Association, 53(4), 272-281.

Kazemi, M., Waalen, J., Morgan, C., \& White, A. (2006). A Profile of Olympic Taekwondo Competitors. Journal of Sports Science and Medicine, 5(CSSI), 114-121.

Koh, J. O., Watkinson, E. J., \& Yoon, Y. J. (2004). Video analysis of head blows leading to concussion in competition taekwondo. Brain Injury, 18(12), 87-96.

Laird, P., \& McLeod, K. (2009). Notational analysis of scoring techniques in competitive men's karate. International Journal of Performance Analysis of Sport, 9, 171-187.

Lee, C., Lee, Y., \& Cheong, C. (2005). A Kinematical analysis of the Taekwondo ap Chagi. En Q. Wang (ed.), Proceedings of the XXIII International Symposium on Biomechanics in Sports (pp. 595597). China Institute of Sport Science, Beijing, China. 
Luk, T. Z., Hong, Y., \& Chu, D. P. K. (2001). Analysis of strategy used in taekwondo competition. En J. R. Blackwell, \& R. H. Sanders (eds.), Proceedings of the XIX International Symposium on Biomechanics in Sports (pp. 166-169). International Society of Biomechanics in Sports, San Francisco, USA.

Lystad, R. P., Pollard, H., \& Graham, P. L. (2009). Epidemiology of injuries in competition taekwondo: A meta-analysis of observational studies. Journal of Science and Medicine in Sport, 12(6), 614-621.

Matsushigue, K. A., Hartmann, K., \& Franchini, E. (2009). Taekwondo: Physiological responses and match analysis. Journal of Strength and Conditioning Research, 23(4), 1112-1117.

Miller, S. A., \& Bartlett, R. M. (1994). Notational analysis of the physical demands of basketball. Journal of Sports Science, 12(2), 181.

Moenig, U. (2011). The evolution of kicking techniques in Taekwondo. Journal of Asian Martial Arts, 20(1), 8-31.

O’Donoghue, P., \& Ingram, B. (2001). A notational analysis of elite tennis strategy. Journal of Sports Sciences, 19, 107-115.

Pieter, F., \& Pieter, W. (1995). Speed and force in selected taekwondo techniques. Biology of Sport, 12(4), 257-266.

Salvador, A., Suay, F., Martinez-Sanchis, S., Simon, V. M., \& Brain, P. F. (1999). Correlating testosterone and fighting in male participants in judo contests. Physiological Behaviour, 68, 205-209.

Santos, V., Franchini, E., \& Lima-Silva, A. (2011). Relationship between attack and skipping in taekwondo contests. Journal of Strength and Conditioning Research, 25(6), 1743-1751.

Serina, E., \& Lieu, D. K. (1991). Thoracic injury potential of basic competition taekwondo kicks. Journal of biomechanics, 24(10), 951-960.

Sterkowicz, S., \& Franchini, E. (2000). Techniques used by judoists during the World and Olympic Tournaments 1995- 1999. Human Movement, 2(2), 23-33.

Sterkowicz, S., Lech, G., Rukasz, W., \& Młynarczyk, J. (2006). Differences in technical and tactical schooling of men and women who practise taekwondo according to the WTF. En W. J. Cynarski, R. M. Kalina, \& K. Obodyński (eds.), 1st World Congress of Combat Sports and Martial Arts Proceedings. Theory, methodology of training and the structure of combat (pp. 27-28). Rzeszów, Poland.

Tang, W. T., Chang, J. S., \& Nien, Y. H. (2007). The kinematics characteristics of preferred and nonpreferred roundhouse kick in elite taekwondo athletes. Journal of Biomechanics, 40(S2), 780.

Thomas, C., Fellingham, G., \& Vehrs, P. (2009). Development of a Notational Analysis System for Selected Soccer Skills of a Women's College Team. Measurement in Physical Education and Exercise Science, 13, 108-113.

Wąsik, J., \& Slęzak, A. (2004). The evaluation of the efficiency of various techniques in Taekwon-do female sparring over $70 \mathrm{~kg}$. En Politechnika Czestochowska (ed.), Sport training in interdisciplinary scientific researches (pp. 320-325). Politechnika Czestochowska, Czestochowska, Poland.

Yujin, Z., \& Zeng, Y. J. (1999). An analysis of Chinese taekwondo team participation of men's 14th and women's 7th World Championships. Journal of Wuahu Institute of Physical Education, 33, 37-39.

Zen-Pin, L., \& Ryder, C. (2001). The Study of Physiological Factors and Performance in Welterweight Taekwondo Athletes. The Sport Journal, 7(2). Extraído desde http://www.thesportjournal.org/article/studyphysiological-factors-and-performancewelterweight-taekwondo-athletes.

World Taekwondo Federation (2012). Competition rules. Extraído desde http://www.wtf.org/wtf eng/site/rules/competition.html.

\section{Datos biográficos de los autores}

Cristina Menescardi Royuela es Licenciada en Ciencias de la Actividad Física y el Deporte (Universidad Católica de Valencia), Máster Universitario en formación en la investigación universitaria en la Universidad Católica de Valencia. También ha participado en diversos proyectos como investigadora colaboradora y tiene 
experiencia en análisis biomecánico del combate y análisis notacional en deportes de equipo y deportes de combate. E-mail: cristina.menescardi@mail.ucv.es.

José Luis Bermejo es Licenciado en Ciencias de la Actividad Física y el Deporte (Universitat de Valencia) y becario de investigación (Universidad Católica de Valencia). Ha participado en diversos proyectos como investigador colaborador y tiene experiencia en análisis biomecánico en deportes de combate y análisis notacional. E-mail: j.l.bermejo@ucv.es.

Carlos Herrero Muria es Graduado en Ciencias de la Actividad Física y el Deporte (Universidad Católica de Valencia). Ha participado en diversos proyectos como investigador colaborador y cuenta con experiencia en análisis biomecánico en deportes de combate y análisis notacional. E-mail: carlos.herrero@mail.ucv.es.

Isaac Estevan Torres (PhD), es profesor en el Grado en Ciencias de la Actividad Física y el Deporte de la Universidad Católica de Valencia. Es autor y coautor de diversos artículos en revistas de impacto nacionales e internacionales sobre biomecánica y ciencias del deporte. Además, es asesor técnico de taekwondo del Comité Español de Deporte Universitario (CEDU) para el Consejo Superior de Deportes. Entre sus responsabilidades, está la organización del Campeonato de España Universitario de Taekwondo. Ha sido deportista de élite de taekwondo, destacando como Campeón de España absoluto (2007), Campeón de España Universitario (2001, 2005, 2006 y 2007), así como cuartofinalista en Campeonatos del Mundo Universitario (2004 y 2006) y Universiadas de Verano (2005 y 2007). E-mail: isaac.estevan@ucv.es.

Raul Landeo, PhD from the University of New South Wales, is a Biomechanics Lecturer at the School of Exercise Science of the Australian Catholic University. He also serves as Sports Education and Development Manager for Taekwondo Australia Inc. Research interests include the tactical and Biomechanical aspects of Combat Sports, 3-D Dynamics and muscle modeling. He is a former elite level Taekwondo athlete and coach. E-mail: raul.landeo@acu.edu.au.

Coral Falcó Pérez (PhD) es profesora en el Grado de Ciencias de la Actividad Física y el Deporte de la Universidad Católica de Valencia. Sus publicaciones cubren un amplio espectro de temas relacionados con el taekwondo en ámbitos científicos como el estudio de la técnica desde un punto de vista cinético y cinemático, análisis de las fuerzas de impacto, análisis del somatotipo, o el estilo de liderazgo. Ha sido deportista de élite, destacando como campeona de la Copa del Mundo (Caiman Islands, 1993) y campeona de Europa (Croazia, 1992).E-mail: coral.falco@ucv.es. 